nephron

Practice
Nephron 2015;129:253-262

DOI: $10.1159 / 000381476$
Received: September 9, 2014

Accepted after revision: March 7, 2015

Published online: April 16, 2015

\title{
Kidney Volume Estimations with Ellipsoid Equations by Magnetic Resonance Imaging in Autosomal Dominant Polycystic Kidney Disease
}

\author{
Eiji Higashihara ${ }^{a}$ b Kikuo Nutahara ${ }^{b}$ Takatsugu Okegawa ${ }^{b}$ Mitsuhiro Tanbo $^{b}$ \\ Hidehiko Harab $^{b}$ Isao Miyazakic Kuninori Kobayasi ${ }^{d}$ Toshiaki Nitatoric ${ }^{c}$ \\ ${ }^{a}$ Department of ADPKD Research, Kyorin University School of Medicine, ${ }^{b}$ Department of Urology, Kyorin University \\ School of Medicine, 'Department of Radiology, Kyorin University School of Medicine, and d Department of Medical \\ Radiological Technology, Faculty of Health Sciences, Kyorin University, Tokyo, Japan
}

\section{Key Words}

Autosomal dominant polycystic kidney disease .

Kidney volume $\cdot$ Ellipsoid volume equation · Magnetic resonance imaging

\section{Abstract \\ Background: Kidney volume (KV) becomes clinically rele- vant in autosomal dominant polycystic kidney disease (ADPKD) management. KV can be conveniently estimated (ceKV) using ellipsoid volume equations with three axes measurements; however, the accuracy and reliability are un- known. Methods: KVs of 347 kidneys in 177 consecutive ADPKD patients were determined with a volumetric method (standard-KV), and ceKV was calculated using six different ellipsoid equations with three axes measurements using magnetic resonance imaging. The inter- and intraobserver reliabilities were analyzed using intraclass correlation coef- ficients (ICCs). Ellipsoid-KVs were obtained by linear regres- sion analysis between standard-KV and ceKVs, and six ellip- soid-KVs were validated with a bootstrap model. Results: The ICCs of intra- and interobserver reliabilities in standard- $\mathrm{KV}$ and axes measurements were highly reliable. All ceKVs underestimated standard-KV and \% differences between ceKV and standard-KV were reduced by ellipsoid-KVs. Boot-}

strap analyses suggested that six ellipsoid-KVs reliably simulated standard-KV. Conclusion: Among six ellipsoid-KVs, ellipsoid- $-\mathrm{KV}_{3}=84+1.01 \times \pi / 24 \times$ Length $\times$ (sum of two width measurements) $)^{2}$ relatively accurately simulated the standard-KV. Kidney volume estimation using ellipsoid equations is reliably applied to clinical management of ADPKD while recognizing wide scattering in the difference between estimated and volumetrically measured kidney volume.

(c) 2015 S. Karger AG, Basel

\section{Introduction}

Autosomal dominant polycystic kidney disease (ADPKD) is a progressive disorder. Kidney function deteriorates and kidney volume (KV) increases progressively. KV has been recognized as a surrogate biomarker of kidney function [1-3]. Recent reports suggest that KV has a prognostic value to predict kidney function deterioration $[4,5]$.

Volumetric quantification of KV based on magnetic resonance imaging (MRI) (standard-KV) is accurate and is regarded as a standard method, but it requires a specialized workstation and time-consuming work [5-8]. The measurement of kidney axes using MRI can be done in

\begin{tabular}{ll}
\hline KARGER 125\% & $\begin{array}{l}\text { (1) 2015 S. Karger AG, Basel } \\
1660-8151 / 15 / 1294-0253 \$ 39.50 / 0 \quad \text { Karger }\end{array}$ \\
$\begin{array}{l}\text { E-Mail karger@karger.com } \\
\text { www.karger.com/nef }\end{array}$ & $\begin{array}{l}\text { This is an Open Access article licensed under the terms of the } \\
\text { Creative Commons Attribution-NonCommercial 3.0 Un- } \\
\text { ported license (CC BY-NC) (www.karger.com/OA-license), } \\
\text { applicable to the online version of the article only. Distribu- } \\
\text { tion permitted for non-commercial purposes only. }\end{array}$
\end{tabular}

Prof. Eiji Higashihara

Department of ADPKD Research, Kyorin University School of Medicine 6-20-2 Shinkawa, Mitaka

Tokyo 181-8611 (Japan)

E-Mail ehigashi@ks.kyorin-u.ac.jp 

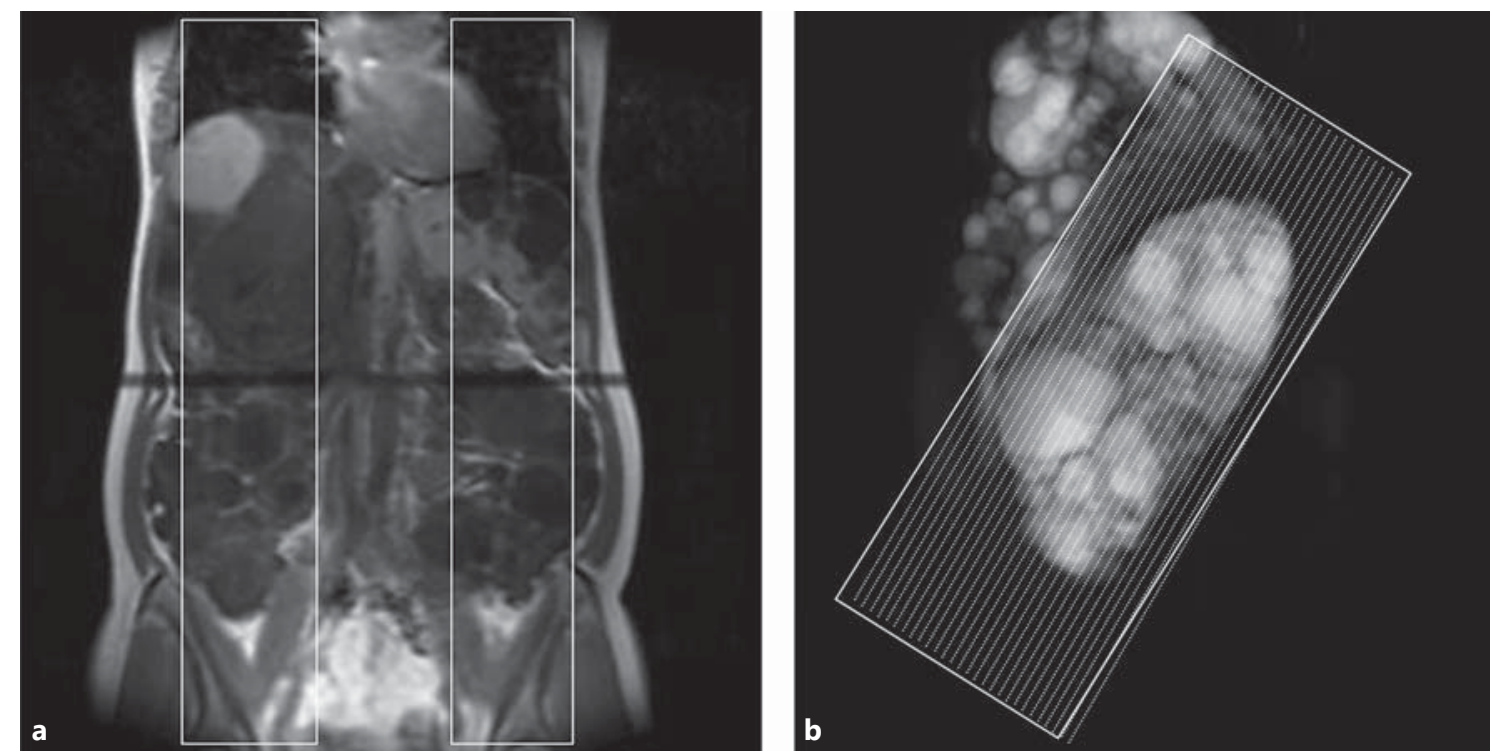

Fig. 1. Monitor coronal image to localize the center portion of the kidneys (a) and tilted coronal images were decided for parallel slicing to the renal long axis (b). Monitor coronal image for positioning two sagittal T2-weighted slices with thickness of 70-100 $\mathrm{mm}$ at the

office practice without software or technical education [5, 9]. The accuracy and reliability of conveniently estimating kidney volume (ceKV) using ellipsoid formulas based on two or three axes measurements on MRI were examined.

\section{Subjects and Methods}

\section{ADPKD Patient Selection}

The MR images and pertinent data were collected consecutively from the data files of 177 ADPKD patients. Standard-KV measurements in ADPKD patients have been performed since 2007 with the same methods of MRI acquisition and volumetric measurement [3]. All patients fulfilled Pei's diagnostic criteria for ADPKD [10]. Only one set of MRI images was extracted from each patient.

The study protocol was approved by an institutional review board (09-56), and the study was conducted in accordance with the guidelines of the Declaration of Helsinki. All participants gave written informed consent to use their clinical data for medical research.

\section{MRI Acquisition}

A non-contrast MRI acquisition protocol used was according to that of Tolvaptan Efficacy and Safety in Management of Autosomal Dominant Polycystic Kidney Disease and Its Outcomes (TEMPO 3/4 study) [11-13]. All images were acquired with the patient in a standard supine position using a 1.5-T scanner. Kidney image acquisition included coronal T2-weighted single-shot fast spin echo images with fat saturation. center of each kidney (a). The sagittal kidney thick image was used to adjust the coronal slice angle parallel to the renal long axis. The tilted T2-weighted coronal images covering the whole kidney area were sequentially obtained with a fixed slice thickness of $4 \mathrm{~mm}$ (b).

Two sagittal T2-weighted single-shot fast spin echo images with slice thicknesses of 70 to $100 \mathrm{~mm}$ were obtained using the socalled 'thick slab' technique (fig. 1a). The axis angle between the kidney and body was determined on the sagittal images, and the coronal slicing was tilted according to the angle (fig. 1b). When the angle was different between the two kidneys, the mean angle was used for sequential slicing. The tilted coronal T2-weighted images covering the entire kidney area were sequentially obtained with a fixed slice thickness of $4 \mathrm{~mm}$ during breath-hold.

\section{Standard-KV Measurement}

All coronal images were transferred to a 3D workstation [3]. Using the signal intensity threshold difference of the renal parenchyma and the background, the kidney border was delineated on each slice and a 3D kidney image was extracted with an automated algorithm [14]. When the border of the kidney was unclear due to factors such as a large polycystic liver or a hemorrhagic cyst (i.e. low signal intensity cysts on T2-weighted images), delineation was manually performed by referring to the T1-weighted images acquired using 3D first field-echo (3DFFE).

The details of image acquisition and image parameters are described in the supplementary material.

\section{MRI Measurement of Renal Length, Width and Depth}

Maximal longitudinal length (L) of the kidney was determined on the T2-weighted tilted coronal slices parallel to the renal long axis (fig. 2a). L was measured obliquely taking into consideration the right-to-left axis rotation. The maximal width (W) was determined perpendicularly to $\mathrm{L}$ on the same slice that $\mathrm{L}$ was localized. In addition to $\mathrm{W}$, a longer width than $\mathrm{W}(\mathrm{WW})$ was surveyed on the other tilted coronal slices. The maximal depth (D) was determined perpendicular to $L$ in a sagittal thick slice (fig. $2 b$ ). 

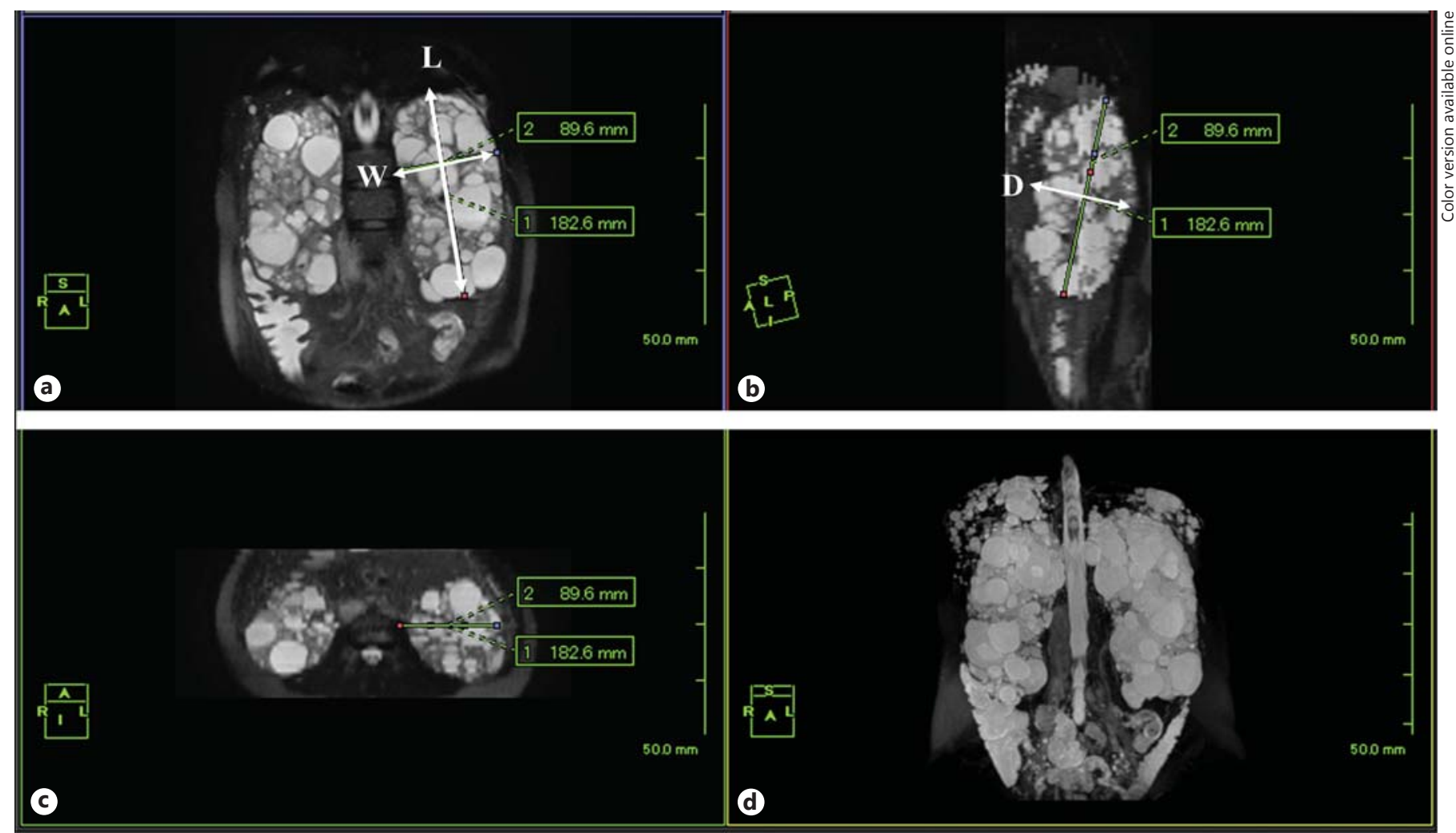

Fig. 2. The actual MR images used for axis measurements. The maximal longitudinal length $(\mathrm{L})$ of the kidney was determined on the T2-weighted tilted coronal slices parallel to the renal long axis (a). L was measured obliquely on taking into consideration the

ceKV with Six Different Ellipsoid Equations

ceKVs derive from the following six equations:

1. $\operatorname{ceKV}_{1}=\pi / 6 \times \mathrm{L} \times \mathrm{W} \times \mathrm{D}$

2. $\mathrm{ceKV}_{2}=\pi / 12 \times \mathrm{L} \times(\mathrm{W}+\mathrm{WW}) \times \mathrm{D}$

3. $\mathrm{ceKV}_{3}=\pi / 24 \times \mathrm{L} \times(\mathrm{W}+\mathrm{WW})^{2}$

4. $\mathrm{ceKV}_{4}=\pi / 54 \times \mathrm{L} \times(\mathrm{W}+\mathrm{WW}+\mathrm{D})^{2}$

5. $\mathrm{ceKV}_{5}=\pi / 6 \times \mathrm{L} \times \mathrm{W}^{2}$

6. $\mathrm{ceKV}_{6}=\pi / 6 \times \mathrm{L} \times \mathrm{WW}^{2}$

These equations were selected based on the basic ellipsoid equations ( 1 and 2 ) and four spheroid equations.

\section{Analysis of Intraobserver and Interobserver Reliability}

Intra- and interobserver reliabilities were examined using an intraclass correlation coefficient (ICC) [15]. Twenty kidneys from ten patients ( 4 men and 6 women, mean age; $50.0 \pm 13.8$, kidney volume; from 232 to $3,131 \mathrm{ml}$ ) were selected randomly to cover a wide range of kidney volumes and were used for ICC analyses for standard-KV and renal axis measurements [16]. The standard-KVs of the twenty kidneys were assessed two times separately by two observers. Three observers measured the L, W, WW and D of 20 kidneys two times independently. All assessments were carried out blindly to their previous measurement results.

Kidney Volume Estimation by MRI in ADPKD right-to-left axis rotation. The maximal width (W) was determined perpendicularly to $\mathrm{L}$ on the same slice that $\mathrm{L}$ was localized. The maximal depth (D) was determined perpendicularly to $L$ in a thick sagittal slice $(\mathbf{b})$.

Statistical Analyses for Linear Regression and ICC

The linear regression equations (ellipsoid-KVs) between standard-KV and ceKV were calculated by linear regression analysis using a full data set $(n=346)$. Variables between men and women were compared by the Welch t test or Mann-Whitney U test. Analyses were performed using JMP (SAS Institute Inc., Cary, N.C., USA).

The Validity Evaluation of the Linear Regression Analyses by Bootstrap Estimation

The internal validity of ellipsoid-KVs was examined by the bootstrap estimation method [17-19]. A two thirds (230) data set was randomly drawn from 346 full data sets and 500 bootstrap repetitions were done with each ellipsoid-KV equation.

\section{Results}

\section{Characteristics of the ADPKD Patients}

Totally, 347 kidney MR images from 177 patients with ADPKD satisfied the MRI acquisition methods (table 1). Two patients had a solitary kidney. Five kidneys were ex- 
Table 1. Demographic characteristics of 177 ADPKD patients selected consecutively from the data file of our institute

\begin{tabular}{|c|c|c|c|c|}
\hline & Total & Men & Women & $\mathrm{p}$ value \\
\hline Number & 177 & 64 & 113 & \\
\hline Age, years & $48.4(15.3)$ & $47.2(14.8)$ & $49.0(15.6)$ & 0.78 \\
\hline $\mathrm{Cr}, \mathrm{mg} / \mathrm{dl}$ & $2.23(2.84)$ & $2.58(2.96)$ & $2.04(2.78)$ & 0.11 \\
\hline $\mathrm{eGFR}, \mathrm{ml} / \mathrm{min} / 1.73 \mathrm{~m}^{2}$ & $55.2(36.7)$ & $53.3(36.0)$ & $56.3(37.2)$ & 0.70 \\
\hline CKD $1(\geq 90)$ & 32 & 13 & 19 & \\
\hline CKD $2(60-89)$ & 47 & 12 & 35 & \\
\hline CKD $3(30-59)$ & 47 & 19 & 28 & 0.49 \\
\hline CKD 4 (15-29) & 21 & 7 & 14 & \\
\hline CKD $5(<15)$ & 30 & 13 & 17 & \\
\hline Height, cm & $163.0(6.4)$ & $172.4(7.1)$ & $157.7(6.0)$ & $<0.0001$ \\
\hline Body weight, kg & $58.8(103)$ & $67.0(10.7)$ & $54.1(10.0)$ & $<0.0001$ \\
\hline Right kidney volume, ml & $969.6(664.3)$ & $1,060.5(627.5)$ & $917.2(684.5)$ & 0.086 \\
\hline Left kidney volume, ml & $1,015.8(650.3)$ & $1,140.3(661.4)$ & $945.3(644.0)$ & 0.0285 \\
\hline Total kidney volume, ml & $1,962.8(1,263.9)$ & $2,158.1(1,188.2)$ & $1,852.1(1,304.5)$ & 0.0618 \\
\hline Ht-TKV, ml/m & $1,203.3(775.6)$ & $1,248.9(677.0)$ & $1,177.4(825.9)$ & 0.278 \\
\hline
\end{tabular}

$\mathrm{Cr}=$ Serum creatinine concentration; GFR = glomerular filtration rate; eGFR = estimated GFR using the Japanese modification of IDMS-MDRD; eGFR $=194 \times \mathrm{Cr}^{-1.094} \times$ age $^{-0.287} \times 0.739$ (if female); MDRD = modification of diet in renal disease; IDMS = isotope dilution mass spectrometry; TKV = total kidney volume measured by volumetric method; Ht-TKV = height-adjusted TKV. p values, comparison between men and women, were analyzed by the Welch t test. Data are mean (SD). Chronic kidney disease (CKD) stage distribution was analyzed by the Mann-Whitney U test.

cluded from the analyses because their long axes were laid close to the horizontal. None of the kidneys were atypical (class $2[5])$.

\section{Intra- and Interobserver Reliability}

The results of intra- and interobserver ICC analyses for axes and volumetric measurements showed that these measurements were reliable and the ICCs were at least above 0.9 (table 2).

Linear Regression Analyses between Standard-KV and ceKV

The correlation coefficients between standard-volume and six ceKVs were equally high (table 3; fig. 3 ). The slopes of the six ceKVs were close to 1.0. The slope of $\mathrm{ceKV}_{3}$ (1.01) was the closest to 1.0. Intercepts ranged from 84.1 to $188.4 \mathrm{ml}$, suggesting that ceKVs underestimated standard-KV. The lowest intercept of 84.1 was obtained with $\mathrm{ceKV}_{3}$.

\section{Validity Evaluation of Ellipsoid-KVs by Bootstrap}

Analysis (table 4)

The slopes and intercepts of ellipsoid-KVs and those of bootstrap estimations were in good agreement, especially in equations (3), (5) and (6). Approximate biases (difference between linear regression result and bootstrap estimation) of the slope and intercept of equation (3) were very small ( 0.0020 and -1.273 , respectively). These bootstrapping results suggest high validity for the ellipsoid volume simulation, especially using ellipsoid $-\mathrm{KV}_{3}$, ellipsoid- $\mathrm{KV}_{5}$ and ellipsoid- $\mathrm{KV}_{6}$.

\section{Consequences of Using Ellipsoid-KV Instead}

Standard-KV

The \% differences in ceKVs and ellipsoid-KVs from the standard-KV are presented in table 5 . The $\%$ difference in ceKV $\mathrm{K}_{3}$ and ellipsoid- $\mathrm{KV}_{3}$ were relatively smaller among the six equations and are illustrated in figure 4 . The percentage difference between ceKV 3 and standardKV exceeded $20 \%$ in 100 of 347 kidneys (28.8\%) and the $\%$ difference exceeded $20 \%$ between ellipsoid- $\mathrm{KV}_{3}$ and standard-KV in 87 kidneys (25.1\%).

\section{Discussion}

The present study showed that polycystic kidney volume can be estimated reliably and relatively accurately by measuring renal axes length using MRI and ellipsoid volume equations [5]. 
Table 2. Intraclass correlation coefficient (ICC) between two measurements within the same observer (intraobserver) and ICC among different observers (interobserver)

\begin{tabular}{|c|c|c|c|c|}
\hline MRI, ellipsoid formula & Length (L) & Width $1(\mathrm{~W})$ & Width 2 (WW) & Depth (D) \\
\hline Intraobserver ICC, observer 1 & 0.999 & 0.970 & 0.990 & 0.996 \\
\hline Intraobserver ICC, observer 2 & 0.998 & 0.982 & 0.989 & 0.974 \\
\hline Inter-three-observers ICC & 0.989 & 0.949 & 0.965 & 0.969 \\
\hline MRI volumetric measurement & Volume & & & \\
\hline Intraobserver ICC, observer 2 & 0.9996 & & & \\
\hline Inter-two-observers ICC & 0.998 & & & \\
\hline
\end{tabular}

$\mathrm{L}=$ Maximal longitudinal length of the kidney on the tilted coronal slice parallel to the kidney long axis; $\mathrm{W}=$ maximum width of the kidney on the same slice on which $\mathrm{L}$ is measured; WW = maximum width of the kidney on any tilted coronal slice parallel to the kidney long axis; $\mathrm{D}=$ depth of the kidney on the sagittal slice.

Table 3. Parameters calculated by linear regression analysis between standard-KV and six conveniently estimated kidney volume (ceKV) equations

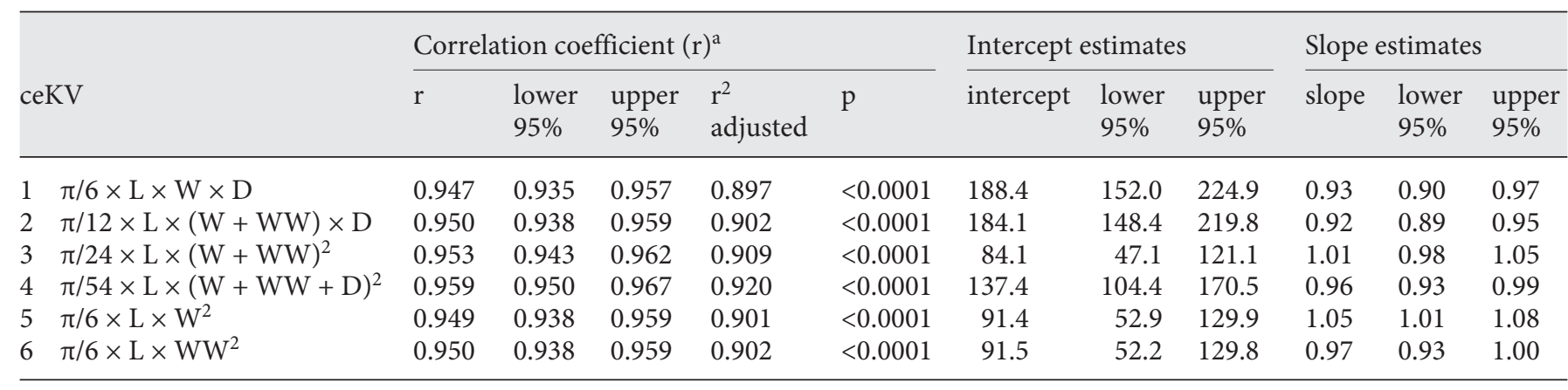

${ }^{a}$ Correlation coefficients of kidney volume calculated by each equation with the kidney volume measured by the volumetric method (standard-KV). L = Maximal longitudinal length of the kidney on the coronal planes sliced parallel to the kidney long axis; $\mathrm{W}=$ maximum width of the kidney on the same plane on which $\mathrm{L}$ is measured; $\mathrm{WW}=$ maximum width of the kidney on any coronal slice parallel to the kidney long axis; $\mathrm{D}=$ depth of the kidney on the sagittal slice. Ellipsoid-KV equations calculated by linear regression analysis for each ceKV. 1) Ellipsoid- $\mathrm{KV}_{1}=188.4+0.93 \times \mathrm{ceKV}_{1}$. 2) Ellipsoid- $\mathrm{KV}_{2}=184.1+0.92 \times \mathrm{ceKV}_{2}$. 3) Ellipsoid- $\mathrm{KV}_{3}=84.1+$ $1.01 \times \mathrm{ceKV}_{3}$. 4) Ellipsoid- $\mathrm{KV}_{4}=137.4+0.96 \times \mathrm{ceKV}_{4}$. 5) Ellipsoid$\left.\mathrm{KV}_{5}=91.4+1.05 \times \mathrm{ceKV}_{5} .6\right)$ Ellipsoid $-\mathrm{KV}_{6}=91.5+0.97 \times \mathrm{ceKV}_{6}$. These equations were selected based on the basic ellipsoid equations (1 and 2) and four spheroid equations (3-6).
An enlarged kidney may change its retroperitoneal position due to a distorted configuration. Its position may passively change frequently due to an enlarged cystic liver. The polycystic kidney tends to decline posteriorly because the space below the kidney is limited by the lumbar and iliac muscles and bone. The variations in anatomical spatial orientation of the kidney affect the axes measurement, but have little effect on volumetric measurement [7, 9].

MRI acquisition adjusting the coronal slice angle in the present study may have mitigated the bias of long re- nal axis measurements. The right-to-left leaning can be compensated for by measuring the longest longitudinal length obliquely on a tilted coronal slice.

The long renal axis can be measured on an unadjusted sagittal slice. However, the length measurement on an unadjusted sagittal slice cannot avoid the influence of right-to-left axis leaning, which is usually in the opposite direction between the two kidneys.

Ultrasonographic measurements of renal axes are less reproducible even in normal kidneys [20, 21]. In 


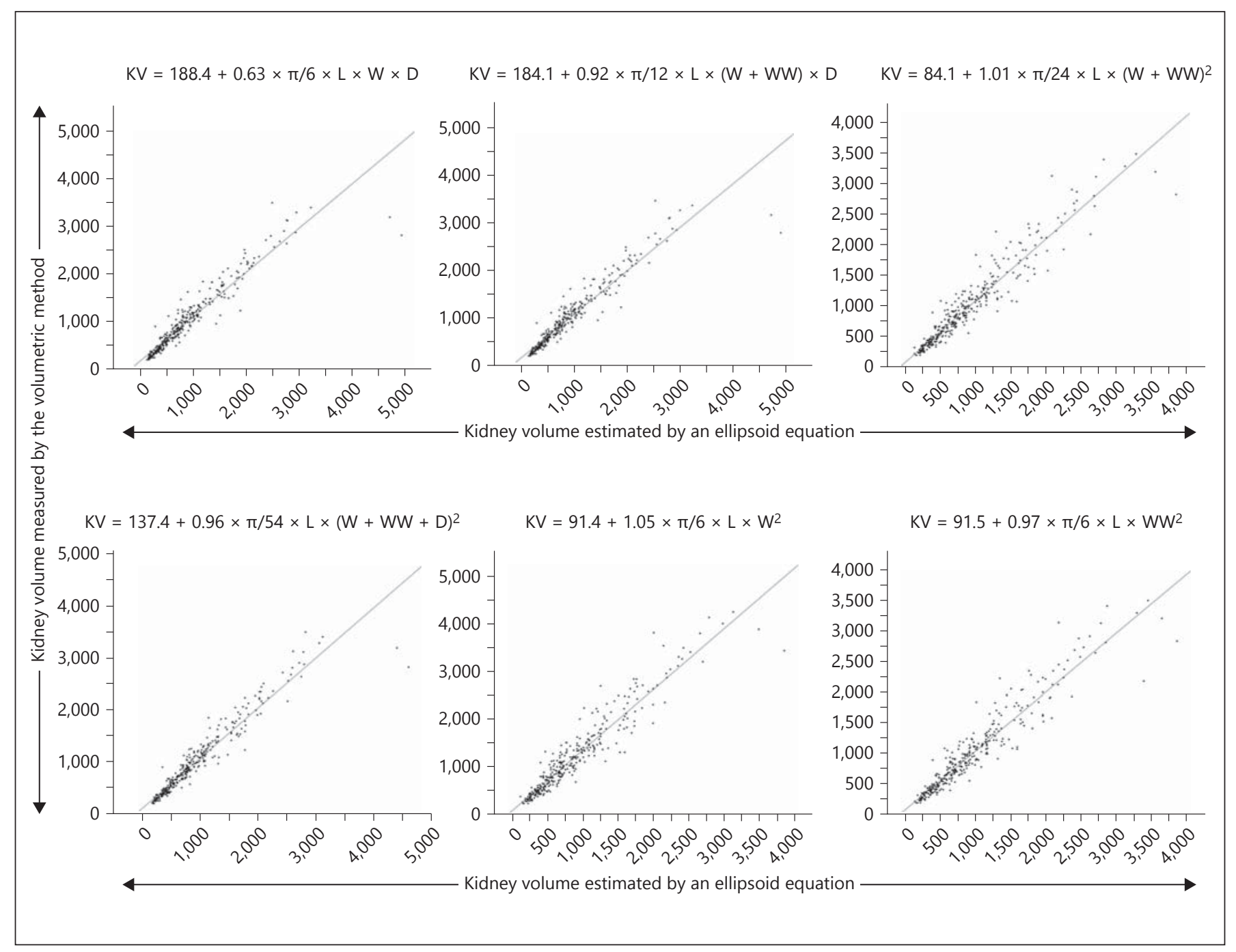

Fig. 3. Six linear regression lines between kidney volume measured by a volumetric method (vertical axis) and that estimated by six ellipsoid equations (horizontal axis). $\mathrm{KV}=$ Kidney volume; $\mathrm{L}=$ maximal longitudinal length of the kidney on the tilted coronal slice parallel to the kidney long axis; $\mathrm{W}=$ maximum width of the kidney on the same slice on which $\mathrm{L}$ is measured; $\mathrm{WW}=$ maximum width of the kidney on any tilted coronal slice parallel to the kidney long axis; $\mathrm{D}=$ depth of the kidney on the sagittal slice.
ADPKD, the interobserver variability was high, and there were about 300 and $700 \mathrm{ml}$ differences in KV measured by ultrasonography and MRI, within the lower and higher volume range, respectively [22]. These results showed that the ultrasonographic KV measurement in ADPKD lacked precision [22, 23].

The KV of patients with ADPKD can be measured by computed tomography (CT). CT volume measurement has similar accuracy and reliability coefficients to MRI [5, $24,25]$. CT can be applied to patients with large kidney and liver volumes. However, the disadvantages of CT include ionized radiation and the use of a nephrotoxic con- trast medium. Given these limitations, CT has a limited role in longitudinal imaging of ADPKD patients [23].

Kidney volumes measured by disc-summation and ellipsoid methods using MRI were compared in 300 normal kidneys [9]. This study reported that an ellipsoid formula $(\pi / 6 \times \mathrm{L} \times \mathrm{W} \times \mathrm{D})$ underestimated renal volume by 17 to $29 \%$ compared with the disc-summation method.

In contrast, in $\mathrm{ADPKD}$, ellipsoid $\mathrm{KV}$ calculated by axes lengths, which were measured by MRI, correlated with standard-KV without systemic underestimation or overestimation [5]. In the present study, we found systemic underestimation of $16.2 \%$ when using the ceKV 
Table 4. Validation results of linear regression analyses by a bootstrap model

\begin{tabular}{|c|c|c|c|c|c|c|}
\hline \multicolumn{7}{|l|}{ Slope estimates } \\
\hline \multicolumn{7}{|l|}{ Linear regression analysis $(\mathrm{n}=346)$} \\
\hline Mean & 0.935 & 0.921 & 1.014 & 0.963 & 1.046 & 0.965 \\
\hline \multicolumn{7}{|l|}{ Bootstrap results $(230 / 346)$} \\
\hline Mean & 0.947 & 0.932 & 1.0155 & 0.972 & 1.048 & 0.968 \\
\hline Approximate bias ${ }^{\mathrm{a}}$ & 0.0119 & 0.0110 & 0.0020 & 0.0083 & 0.0020 & 0.0029 \\
\hline Approximate standard error & 0.074 & 0.070 & 0.0386 & 0.062 & 0.0433 & 0.0399 \\
\hline Lower confidence limit ${ }^{\mathrm{b}}$ & 0.777 & 0.773 & 0.936 & 0.834 & 0.959 & 0.884 \\
\hline Upper confidence limit ${ }^{\mathrm{b}}$ & 1.069 & 1.047 & 1.0873 & 1.076 & 1.129 & 1.041 \\
\hline Linear regression analysis $(\mathrm{n}=3$ & & & & & & \\
\hline Mean & 188.427 & 184.099 & 84.109 & 137.440 & 91.418 & 91.548 \\
\hline Lower 95\% & 152.004 & 143.378 & 47.113 & 104.364 & 52.942 & 53.264 \\
\hline Upper 95\% & 224.850 & 219.821 & 121.105 & 170.516 & 129.894 & 129.832 \\
\hline \multicolumn{7}{|l|}{ Bootstrap results $(230 / 346)$} \\
\hline Mean & 179.391 & 175.736 & 82.836 & 131.092 & 89.867 & 89.715 \\
\hline Approximate bias ${ }^{\mathrm{a}}$ & -9.039 & -8.364 & -1.273 & -6.348 & -1.551 & -1.833 \\
\hline Approximate standard error & 55.253 & 52.745 & 28.198 & 46.638 & 31.138 & 29.825 \\
\hline Lower confidence limit ${ }^{\mathrm{b}}$ & 89.175 & 89.084 & 30.114 & 52.379 & 31.939 & 34.925 \\
\hline Upper confidence limit ${ }^{\mathrm{b}}$ & 305.764 & 295.842 & 140.649 & 235.197 & 153.998 & 151.838 \\
\hline Number larger than mean ${ }^{c}$ & $226 / 500$ & $227 / 500$ & $237 / 500$ & $230 / 500$ & $229 / 500$ & $237 / 500$ \\
\hline p value $^{\mathrm{d}}$ & 0.452 & 0.454 & 0.474 & 0.460 & 0.458 & 0.474 \\
\hline
\end{tabular}

Linear regression analyses were performed using 346 full data sets. Bootstrap significance testings were performed by repeating 500 times on 230 data sets randomly sampled from 346 full data sets. ${ }^{a}$ Approximate bias is the difference between linear regression analysis using full data sets and bootstrap estimation. ${ }^{\mathrm{b}}$ Approxi- mate lower and upper confidence limits. ${ }^{\mathrm{c}}$ Number larger than the mean calculated by linear regression analysis of a full data set. ${ }^{d} p$ value of significant difference between linear regression analysis and bootstrap results.

Table 5. The \% differences in kidney volume estimated by ellipsoid formulas from the kidney volume measured by the volumetric method

\begin{tabular}{|c|c|c|c|}
\hline ceKV & $\begin{array}{l}\% \text { differences } \\
\text { from standard-KV }\end{array}$ & Ellipsoid-KV & $\begin{array}{l}\% \text { differences } \\
\text { from standard-KV }\end{array}$ \\
\hline $\operatorname{ceKV}_{1}=\pi / 6 \times \mathrm{L} \times \mathrm{W} \times \mathrm{D}$ & $-16.2 \pm 15.3$ & ellipsoid- $\mathrm{KV}_{1}=188.4+0.93 \times \mathrm{ceKV}_{1}$ & $6.3 \pm 20.1$ \\
\hline $\operatorname{ceKV} V_{2}=\pi / 12 \times \mathrm{L} \times(\mathrm{W}+\mathrm{WW}) \times \mathrm{D}$ & $-14.5 \pm 15.3$ & ellipsoid- $\mathrm{KV}_{2}=184.1+0.82 \times \mathrm{ceKV}_{2}$ & $-2.2 \pm 18.9$ \\
\hline $\mathrm{ceKV}_{3}=\pi / 24 \times \mathrm{L} \times(\mathrm{W}+\mathrm{WW})^{2}$ & $-9.5 \pm 16.3$ & ellipsoid- $\mathrm{KV}_{3}=84.1+1.01 \times \mathrm{ceKV}_{3}$ & $4.1 \pm 18.4$ \\
\hline $\operatorname{ceKV}_{4}=\pi / 54 \times \mathrm{L} \times(\mathrm{W}+\mathrm{WW}+\mathrm{D})^{2}$ & $-12.5 \pm 13.8$ & ellipsoid- $\mathrm{KV}_{4}=137.4+0.96 \times \mathrm{ceKV}_{4}$ & $4.7 \pm 16.8$ \\
\hline $\operatorname{ceKV}_{5}=\pi / 6 \times \mathrm{L} \times \mathrm{W}^{2}$ & $-12.9 \pm 16.7$ & ellipsoid- $\mathrm{KV}_{5}=91.4+1.05 \times \mathrm{ceKV}_{5}$ & $5.3 \pm 19.8$ \\
\hline $\mathrm{ceKV}_{6}=\pi / 6 \times \mathrm{L} \times \mathrm{WW}^{2}$ & $-5.9 \pm 17.2$ & ellipsoid- $\mathrm{KV}_{6}=91.5+0.97 \times \mathrm{ceKV}_{6}$ & $5.1 \pm 18.7$ \\
\hline
\end{tabular}

Data are mean \pm standard deviation. ceKV $=$ Conveniently estimated kidney volume using ellipsoid equation; ellipsoid-KV = linear regression equation calculated between standard-KV and ceKV; $\mathrm{L}=$ maximal longitudinal length of the kidney on the coro- nal planes sliced parallel to the kidney long axis; $\mathrm{W}=$ maximum width of the kidney on the same plane on which $\mathrm{L}$ is measured; $\mathrm{WW}=$ maximum width of the kidney on any coronal slice parallel to the kidney long axis; $\mathrm{D}=$ depth of the kidney on the sagittal slice. 


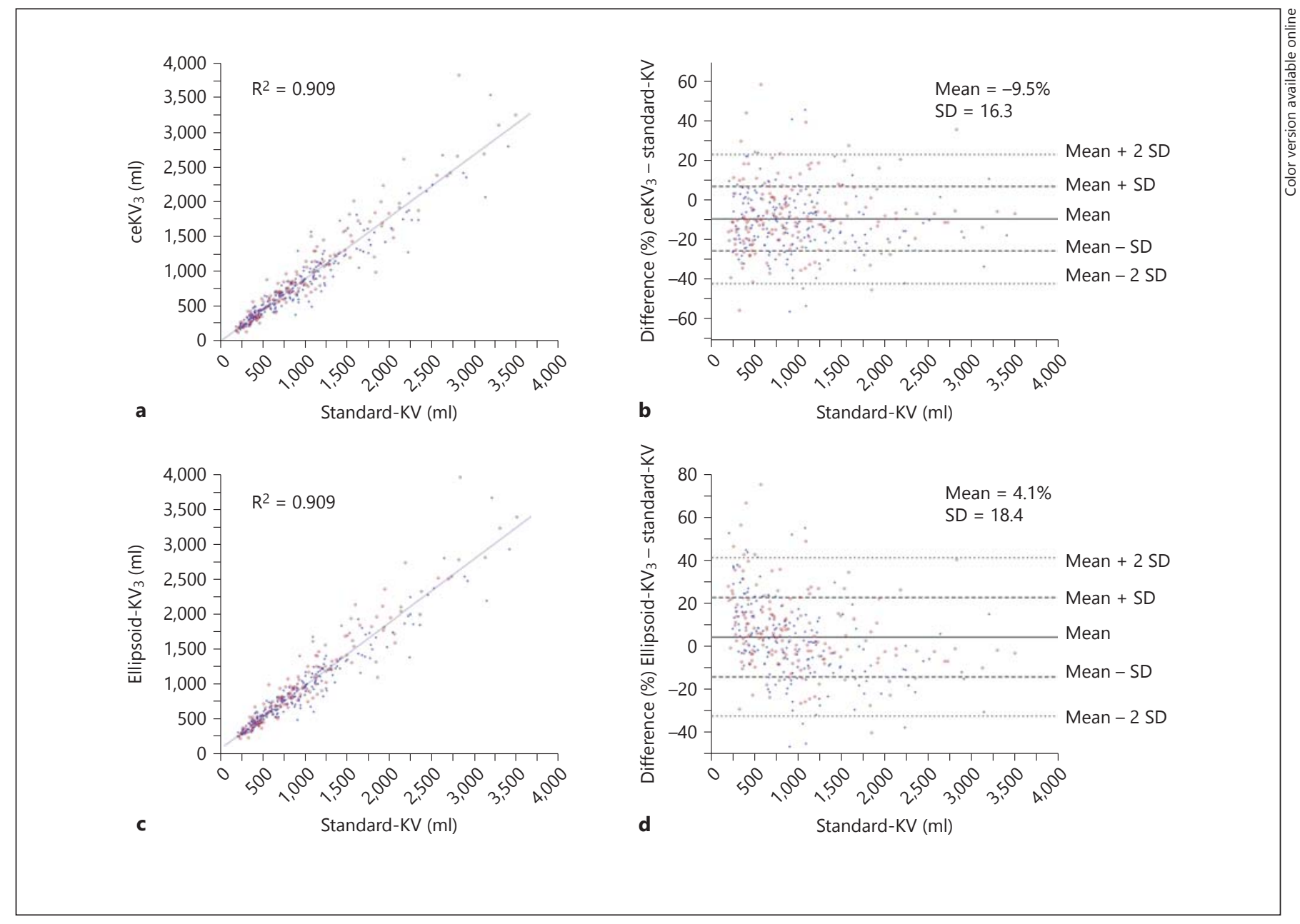

Fig. 4. Correlations between $\mathrm{ceKV}_{3}(\mathbf{a})$ and ellipsoid- $\mathrm{KV}_{3}$ (c) with standard-KV are highly significant. In 100 patients (28.8\%) and 87 patients $(25.1 \%)$, the percent differences from standard-KV ex-

formula (table 5). The long axes measured in sagittal views in the reported study [5] may have been shorter than those measured in the present study, because the longest length could be measured obliquely on a tilted coronal slice. The width and depth measured on the axial (transverse) image [5] could be longer than the actual lengths, because the longitudinal axis of the kidney is tilted anteroposteriorly and/or laterally. Therefore, the discrepancy between this and previous reports may be explained by bias in axis length measurement. Another possibility for this discrepancy may have been the difference in standard-KV measurement.

The percent differences between ellipsoid-KVs and standard-KV were not very large between ellipsoid and spheroid estimations (table 5). This observation suggests ceeded $20 \%$ in ceKV $\mathrm{KV}_{3}(\mathbf{b})$ and ellipsoid- $\mathrm{KV}_{3}(\mathbf{d})$, respectively. Open red circles and closed blue circles indicate the right and left kidney, respectively. that kidney configuration of $\mathrm{ADPKD}$ is close to spheroid (width and depth are about equal) compared to a normal kidney.

The percent difference between standard-KV and ellipsoid- $\mathrm{KV}_{3}$ exceeded $20 \%$ in $25.1 \%$ of all cases (fig. $4 \mathrm{~d}$ ). This percentage was smaller than that between standard$\mathrm{KV}$ and $\mathrm{ceKV}_{3}$ (28.8\%; fig. $4 \mathrm{~b}$ ), but larger than the $5.9 \%$ reported by Irazabal and co-authors [5]. Since results of intra- and interobserver ICC analyses for axes and volumetric measurements and number of observers who participated in axes and volumetric measurements were not reported (5), the reasons for the differences between the two reports are unclear. The wide scatter in measuring kidney axes (table 2) may have resulted in a wide distribution in the difference between standard-KV and ellipsoid- 
KV (fig. 4). Kidney volume estimation using ellipsoid equations yields substantial scatter from standard-KV, and we should be aware of this limitation whenever we use this ellipsoid estimation method.

One limitation of this study is the lack of a direct comparison of axes measurements. The MRI slices for three axes measurements were acquired with different methods from a previous report [5] and this study (fig. 2a). The difference in accuracy between the two methods is difficult to compare. However, the more important message of the present study is the recognition of a relatively wide scatter of ellipsoid kidney volume from volumetrically measured kidney volume, even though there is a high correlation between the two volume measurements (fig. 4).

In conclusion, among six ellipsoid equations examined, a kidney volume estimation equation using axes ( $\mathrm{L}$,
$\mathrm{W}$ and WW) measurement on tilted coronal MRI slices parallel to the renal long axis, kidney volume $(\mathrm{ml})=84+$ $1.01 \times \pi / 24 \times \mathrm{L} \times(\mathrm{W}+\mathrm{WW})^{2}$, was stably close to the volume measured by the volumetric method. This method and equation provide a convenient and relatively accurate estimation of kidney volume in the clinical management of ADPKD when the results are interpreted cautiously.

\section{Disclosure Statement}

This study was supported in part by a Grant-in-Aid for Progressive Renal Diseases Research from the Ministry of Health, Labor and Welfare of Japan. E.H. acknowledges grant support from Otsuka, and has participated on advisory boards for Otsuka and K.N. has received research grants from Otsuka, Takeda, and Daiichi Sankyo.

\section{References}

1 Grantham JJ, Torres VE, Chapman AB, Guay-Woodford LM, Bae KT, King BF Jr, Wetzel LH, Baumgarten DA, Kenney PJ, Harris PC, Klahr S, Bennett WM, Hirschman GN, Meyers CM, Zhang X, Zhu F, Miller JP; CRISP Investigators: Volume progression in polycystic kidney disease. N Engl J Med 2006;354: 2122-2130.

2 Harris PC, Bae KT, Rossetti S, Torres VE, Grantham JJ, Chapman AB, Guay-Woodford LM, King BF, Wetzel LH, Baumgarten DA, Kenney PJ, Consugar M, Klahr S, Bennett WM, Meyers CM, Zhang QJ, Thompson PA, Zhu F, Miller JP: Cyst number but not the rate of cystic growth is associated with the mutated gene in autosomal dominant polycystic kidney disease. J Am Soc Nephrol 2006; 17:3013-3019.

3 Higashihara E, Nutahara K, Okegawa T, Shishido T, Tanbo M, Kobayasi K, Nitadori T: Kidney volume and function in autosomal dominant polycystic kidney disease. Clin Exp Nephrol 2014;18:157-165.

4 Chapman AB, Bost JE, Torres VE, GuayWoodford L, Bae KT, Landsittel D, Li J, King BF, Martin D, Wetzel LH, Lockhart ME, Harris PC, Moxey-Mims M, Flessner M, Bennett WM, Grantham JJ: Kidney volume and functional outcomes in autosomal dominant polycystic kidney disease. Clin J Am Soc Nephrol 2012;7:479-486.

5 Irazabal MV, Rangel LJ, Bergstralh EJ, Osborn SL, Harmon AJ, Sundsbak JL, Bae KT, Chapman AB, Grantham JJ, Mrug M, Hogan MC, El-Zoghby ZM, Harris PC, Erickson BJ, King BF, Torres VE; CRISP Investigators: Imaging classification of autosomal dominant polycystic kidney disease: a simple model for selecting patients for clinical trials. J Am Soc Nephrol 2015;26:160-172.
6 Bae KT, Commean PK, Lee J: Volumetric measurement of renal cysts and parenchyma using MRI: phantoms and patients with polycystic kidney disease. J Comput Assist Tomogr 2000;24:614-619.

-7 Bae KT, Tao C, Zhu F, Bost JE, Chapman AB, Grantham JJ, Torres VE, Guay-Woodford LM, Meyers CM, Bennett WM; Consortium for Radiologic Imaging Studies Polycystic Kidney Disease: MRI-based kidney volume measurements in ADPKD: reliability and effect of gadolinium enhancement. Clin J Am Soc Nephrol 2009;4:719-725.

$\checkmark 8$ Mignani R, Corsi C, De Marco M, Caiani EG, Santucci G, Cavagna E, Severi S, Cagnoli L: Assessment of kidney volume in polycystic kidney disease using magnetic resonance imaging without contrast medium. Am J Nephrol 2011;33:176-184.

$\checkmark$ Cheong B, Muthupillai R, Rubin MF, Flamm SD: Normal values for renal length and volume as measured by magnetic resonance imaging. Clin J Am Soc Nephrol 2007;2:38-45.

10 Pei Y, Obaji J, Dupuis A, Paterson AD, Magistroni R, Dicks E, Parfrey P, Cramer B, Coto E, Torra R, San Millan JL, Gibson R, Breuning M, Peters D, Ravine D: Unified criteria for ultrasonographic diagnosis of ADPKD. J Am Soc Nephrol 2009;20:205-212.

11 Torres VE, Meijer E, Bae KT, Chapman AB, Devuyst O, Gansevoort RT, Grantham JJ, Higashihara E, Perrone RD, Krasa HB, Ouyang JJ, Czerwiec FS: Rationale and design of the TEMPO (Tolvaptan Efficacy and Safety in Management of Autosomal Dominant Polycystic Kidney Disease and its Outcomes) 3-4 Study. Am J Kidney Dis 2011;57:692699.
12 Higashihara E, Torres VE, Chapman AB, Grantham JJ, Bae K, Watnick TJ, Horie S, Nutahara K, Ouyang J, Krasa HB, Czerwiec FS; TEMPOFormula and 156-05-002 Study Investigators: Tolvaptan in autosomal dominant polycystic kidney disease: three years' experience. Clin J Am Soc Nephrol 2011;6: 2499-2507.

13 Torres VE, Chapman AB, Devuyst O, Gansevoort RT, Grantham JJ, Higashihara E, Perrone RD, Krasa HB, Ouyang J, Czerwiec FS; TEMPO 3:4 Trial Investigators: Tolvaptan in patients with autosomal dominant polycystic kidney disease. N Engl J Med 2012;367:24072418.

14 Lee YR, Lee KB: Reliability of magnetic resonance imaging for measuring the volumetric indices in autosomal-dominant polycystic kidney disease: correlation with hypertension and renal function. Nephron Clin Pract 2006; 103:c173-c180

15 Shrout PE, Fleiss JL: Intraclass correlations: uses in assessing rater reliability. Psychol Bull 1979;86:420-428.

16 Saranadasa H: The square root of $\mathrm{N}$ plus one sampling rule. Pharmaceutical Technology, 2003, pp 50-62.

-17 Forman DE, Butler J, Wang Y, Abraham WT, O'Connor CM, Gottlieb SS, Loh E, Massie BM, Rich MW, Stevenson LW, Young JB, Krumholz HM: Incidence, predictors at admission, and impact of worsening renal function among patients hospitalized with heart failure. J Am Coll Cardiol 2004;43:61-67.

-18 Steyerberg EW, Bleeker SE, Moll HA, Grobbee DE, Moons KG: Internal and external validation of predictive models: a simulation study of bias and precision in small samples. J Clin Epidemiol 2003;56:441-447. 
19 Bae KT, Tao C, Wang J, Kaya D, Wu Z, Bae JT, Chapman AB, Torres VE, Grantham JJ, Mrug M, Bennett WM, Flessner MF, Landsittel DP; Consortium for Radiologic Imaging Studies of Polycystic Kidney Disease (CRISP): Novel approach to estimate kidney and cyst volumes using mid-slice magnetic resonance images in polycystic kidney disease. Am J Nephrol 2013;38:333-341.

20 Solvig J, Ekberg H, Hansen F, Brunkvall J, Länne T: Accuracy of noninvasive ultrasonic volume measurements on human kidney transplants. Presentation of a new formula. Nephron 1998;80:188-193.
21 Bakker J, Olree M, Kaatee R, de Lange EE, Moons KG, Beutler JJ, Beek FJ: Renal volume measurements: accuracy and repeatability of US compared with that of MR imaging. Radiology 1999;211:623-628.

22 O'Neill WC, Robbin ML, Bae KT, Grantham JJ, Chapman AB, Guay-Woodford LM, Torres VE, King BF, Wetzel LH, Thompson PA, Miller JP: Sonographic assessment of the severity and progression of autosomal dominant polycystic kidney disease: the Consortium of Renal Imaging Studies in Polycystic Kidney Disease (CRISP). Am J Kidney Dis 2005;46:1058-1064.
3 Chapman AB, Wei W: Imaging approaches to patients with polycystic kidney disease. Semin Nephrol 2011;31:237-244.

24 Sise C, Kusaka M, Wetzel LH, Winklhofer F, Cowley BD, Cook LT, Gordon M, Grantham JJ: Volumetric determination of progression in autosomal dominant polycystic kidney disease by computed tomography. Kidney Int 2000;58:2492-2501.

25 Antiga L, Piccinelli M, Fasolini G, Ene-Iordache B, Ondei P, Bruno S, Remuzzi G, Remuzzi A: Computed tomography evaluation of autosomal dominant polycystic kidney disease progression: a progress report. Clin J Am Soc Nephrol 2006;1:754-760. 